\title{
Impact and cultural acceptance of the Narrative Exposure Therapy in the aftermath of a natural disaster in Burundi
}

Anselm Crombach ${ }^{1,2,3,4^{*}}$ (D) and Sebastian Siehl ${ }^{4,5,6}$

\begin{abstract}
Background: In the aftermath of natural disasters, affected populations are at risk of suffering from trauma-related mental health disorders such as posttraumatic stress disorder (PTSD) or depression. Particularly in poor post-conflict regions, these mental disorders have the potential to impair the ability of individuals to move on with their lives. We aimed to evaluate the feasibility, cultural acceptance, and effect of a trauma-focused psychotherapy, Narrative Exposure Therapy (NET), in the aftermath of a flood disaster in Burundi.
\end{abstract}

Methods: Fifty-one individuals who were living in emergency camps overseen by the Burundian Red Cross in the aftermath of a flood disaster, and who had lost homes and close relatives, were invited to participate in semistructured diagnostic interviews. Trained Burundian psychology students conducted these interviews, and six sessions of NET were offered to the 15 individuals most affected by trauma-related symptoms. An additional group of psychology students, blind to the treatment conditions, conducted three and 9 months follow-ups with them including also 25 participants who had reported significant but less severe trauma-related symptoms, assessing mental health symptoms, acceptance of NET, stigmatization due to trauma symptoms, and participants' economic well-being.

Results: Between baseline and 9-months post-intervention assessment, symptoms of PTSD (Hedges' $g=3.44$ ) and depression (Hedges' $g=1.88$ ) improved significantly within participants who received NET and within those who received no treatment (Hedges ${ }^{\prime} g_{\text {PTSD }}=2.55$; Hedges ${ }^{\prime} g_{\text {depression }}=0.72$ ). Furthermore, those who received NET felt less stigmatized by their participation in the intervention than by the trauma-related mental health symptoms they experienced. Overall, participants reported that they would be willing to forego as much as 1 month's worth of income in exchange for receiving trauma-focused interventions in the months following the disaster.

Conclusions: Individuals severely affected by trauma-related mental health symptoms might benefit significantly from NET in the aftermath of natural disasters, while less affected individuals seem to recover spontaneously. Despite significant challenges conducting NET in emergency camps in the aftermath of natural disaster in a post-conflict country, such interventions are feasible, appreciated and might have long-lasting impacts on the lives of survivors if conducted with due respect to participants' privacy.

Trial registration: UKCR2014, the 19.06.2014, retrospectively registered.

Keywords: Narrative Exposure Therapy, Natural disaster, post-conflict, Trauma-focused interventions, Psychological assistance, Burundi

\footnotetext{
* Correspondence: anselm.crombach@uni-konstanz.de

${ }^{1}$ Department of Psychology, University of Konstanz, Konstanz, Germany

${ }^{2}$ Department of Psychology, University Lumière of Bujumbura, Bujumbura,

Burundi

Full list of author information is available at the end of the article
}

(c) The Author(s). 2018 Open Access This article is distributed under the terms of the Creative Commons Attribution 4.0 International License (http://creativecommons.org/licenses/by/4.0/), which permits unrestricted use, distribution, and reproduction in any medium, provided you give appropriate credit to the original author(s) and the source, provide a link to the Creative Commons license, and indicate if changes were made. The Creative Commons Public Domain Dedication waiver (http://creativecommons.org/publicdomain/zero/1.0/) applies to the data made available in this article, unless otherwise stated. 


\section{Background}

In the aftermath of natural and human induced disasters, affected individuals struggle to come to terms with the often-horrifying consequences. Particularly in low-resource crisis and post-conflict regions, the path to recovery is often threatened by economic loss, disrupted community or family systems, and health impairments. Gulliver, Zimering, Carpenter, Giardina, \& Farrar [1] describe a disaster "as a natural or man-made event, that negatively affects life, property, livelihood ..." (p. 25) and which leads to an increased incidence and relapse of mental health disorders. Furthermore, the life-threatening nature of the disaster, the loss of loved ones, and, in some cases, the irreversible physical impairment of affected individuals further contributes to the risk of developing mental health disorders. Such disorders might prevent victims from benefiting sustainably from material aid that is often provided as part of organized relief efforts. In their extensive review, Galea, Nandi, \& Vlahov [2] describe posttraumatic stress disorder (PTSD) as the most frequently occurring psychological disorder appearing after the experience of a natural disaster, with prevalence rates ranging from 5 to $60 \%$, with higher rates in areas more severely affected by the disaster. According to Galea et al. [2] the strongest predictor for developing symptoms of PTSD is the extent of exposure to the disaster, with higher risks for individuals with high exposure. In addition, research on the building block effect, i.e., a dose-response relationship between the number of experienced traumatic event types and PTSD symptoms, emphasizes that prior traumatic experiences have a severe impact on mental health and substantially increase the risk of suffering from trauma-related mental disorders (e.g., [3, 4]). Indeed, in the aftermath of the 2004 tsunami, a study conducted in Sri Lanka identified previous traumatic exposure, severity of exposure to the natural disaster, and loss of family members as significant predictors of PTSD symptoms in children, and consequently found elevated prevalence rates in violence-affected crisis regions compared to more stable regions [5].

The devastating impact of mental health disorders has been increasingly recognized as a crucial risk factor potentially causing long-term human misery and impairment in health and economic productivity in low-income countries [6]. Even though mental health disorders have been identified as a major obstacle to successful recovery, the best way of responding to psychological needs in the aftermath of humanitarian disasters in crisis regions has been subject to highly controversial discussions in recent years. Experts disagreed strongly regarding the implementation of psychotherapeutic interventions to address PTSD. Some have argued that the concept of PTSD is culture-bound and irrelevant outside the context of Western cultures. They stressed that attaching diagnoses and offering treatment might be stigmatizing, and might not sufficiently reflect the emotional suffering and daily worries of individuals in such contexts [7]. Those experts argued that reducing daily stressors, such as financial hardship or barriers to resettlement [8], through psychosocial interventions would be the key to address mental health issues in these circumstances [9]. Furthermore, they emphasized the prominent role of daily stressors in predicting and maintaining mental distress $[8,10]$.

Others argued, however, that correlations between a low socio-economic status (SES) and increased vulnerability for mental disorders, such as symptoms of depression or PTSD, are at least partially explained by the impaired functionality of affected individuals and entire communities. For instance, a study with former child soldiers suggested that symptoms of PTSD are associated with a reduced openness to reconciliation and elevated feelings of revenge [11], thereby impairing trust and collaboration in the community. Furthermore, research indicates that the degree to which potential daily stressors are perceived as stressful depends on the mental health condition of an individual, as a bias towards a negative evaluation and interpretation of a situation is a core element of anxiety disorders and depression [12]. For instance, traumatized individuals also feel threatened more easily and hence might perceive daily struggles as more stressful and intimidating than individuals not suffering from trauma-related disorders. Furthermore, daily stressors are likely to trigger feelings of fear and helplessness, which are then exacerbated by feelings, cognitions, and interoceptive impressions related to previous traumatic experiences $[13,14]$. Therefore, many clinicians emphasize the necessity of addressing trauma-related disorders with evidence-based methods that are evaluated in the specific context in which they are being utilized and can be applied by lay-counsellors instead of aiming to stabilize perpetually unstable environments [7, 15].

Narrative Exposure Therapy (NET) has been developed as a standardized, short-term treatment of PTSD for survivors of war, domestic violence, torture, and natural disaster [16]. NET embeds the principles of testimony therapy [17], prolonged exposure therapy [18], cognitive-behavioural and client-centred psychotherapy into recent findings of neuro-traumatology. Extensive research demonstrated the effectiveness of NET in a wide range of contexts and populations with symptoms of PTSD and depression. Randomized Controlled Trial studies (RCTs) revealed positive treatment effects for adults and children who suffer from mental illness after the experience of multiple traumatic events [19-22], including natural disasters [23, 24]. NET has also been proven effective in a short form with only 4 to 8 sessions, making it a valuable tool in insecure and volatile environments such as in the aftermath of a flood disaster or within post-conflict countries [21, 25]. Such efficacy in unstable situations is compounded by the successful 
dissemination of NET to local laypersons, which has been shown in several studies $[25,26]$. A similar reduction of symptoms in patients with PTSD was found in studies focusing more generally on exposure therapies outside of NET, although those participants received therapy provided by a highly experienced therapist or a trained local health worker [27-29].

Several reviews and meta-analyses provide evidence of the efficacy of NET in a broad range of cultures such as the Middle East, Central- and North Africa as well as Europe [25, 30-32]. Symptoms of PTSD were significantly reduced in all of the studies reviewed with moderate to high effect sizes. In general, the symptom severity continued to decline with longer time periods between the completion of NET and the follow-up. This continuous symptom reduction might be explained by long-term reorganization of memories and neuroplastic changes in the brain following completion of therapy $[13,33]$. However, it has never been attempted to assess quantitatively the degree to which populations in African post-conflict countries value a trauma-focused intervention such as NET as an improvement of their lives. Assessing the value which affected populations attribute to these interventions seems particularly important in the aftermath of acute emergencies, including natural disasters. Such valuations may serve as a guide to institutions financing emergency responses in how to invest their resources regarding material and psycho-social assistance. An approach assessing acceptance is Willingness to Pay (WTP). WTP is defined as the maximum amount of money that an individual is willing to sacrifice in order to get a certain product or service [34]. Although, WTP was originally used for cost-effectiveness analyses, it can be successfully applied in evaluating treatments of PTSD [35] and depression [36]. Unützer et al. [37] concluded that WTP can be used to assess the value of treatments and reported in a sample of 615 American depressed primary care patients an average WTP of $9 \%$ of the participants' monthly household income.

Another obstacle for psychological assistance that has to be taken into account in the aftermath of disasters is stigmatization. Goffman ([38]; pp.4-5) defines social stigma as "the phenomenon whereby an individual with an attribute is deeply discredited by his/ her society and is rejected as a result of the attribute". Read, Haslam, Sayce, \& Davies [39] indicated in their review that stigma might be related to biogenetic causal theories. The word "illness" for example triggers the perception of danger and unpredictability. A natural reaction that follows is fear and the desire for social distance. The result is that clients often face social rejection, dislike, or devaluation by others. Moreover, the belief that symptoms are self-inflicted [40] is widespread. Social stigmatization and isolation seems to be a phenomenon arising independent of cultural background [41] and is also found in individuals with symptoms of PTSD [42]. Hence social stigma can also occur by seeking treatment. Often people then decide not to seek adequate treatment, refrain from fully participating, or drop out [43]. Furthermore, research indicates that social disapproval impairs the recovery from trauma-related symptoms [44-46].

Burundi is a small country in Eastern Africa that has served as a battlefield for two ethnically-driven civil wars during the past 50 years. These civil wars, coupled with other political challenges, have served to decimate the country's ability to cope with the effects of their frequent natural disasters. In February 2014, heavy rainfall flooded several districts of the capital of Burundi, Bujumbura. The resulting floods destroyed the livelihood of over 12,500 people, and killed at least 64 people, most of whom were children under the age of 10 [47]. In the wake of these events, we decided to conduct a feasibility trial regarding the implementation of NET as a trauma-focused intervention in the aftermath of a natural disaster in a post-conflict setting. We assumed that individuals suffering severely from PTSD symptoms in particular might benefit from such an intervention to prevent chronicity of symptoms, while less affected individuals might recover spontaneously once their living conditions became more stable. In addition to the efficacy of NET regarding mental health improvements, we also aimed to assess the fictional monetary value the survivors would attribute to receiving this intervention compared to their SES, and if they would feel more stigmatized by the treatment than by their mental health symptoms.

\section{Methods}

\section{Participants}

Initially, the project started as humanitarian aid project, assisting the Burundian Red Cross in three emergency camps established in response to the flood disaster in February 2014 in Bujumbura. The emergency camps were located in the districts Kinama, Kamenge and Buterere. Burundian Red Cross volunteers working in these camps identified 51 individuals who they considered to be severely affected by the disaster for the mental health experts of our team. Apart from their personal impressions of which survivors might need psychological assistance, they were encouraged to include individuals who had lost a close relative during the disaster, or individuals who were known to be having trouble sleeping, waking up screaming at night or reporting nightmares, and individuals who were socially isolated and did not engage with others in the close camp environments. During the initial diagnostic interviews, we found that 40 individuals suffered significantly from trauma-related mental health symptoms, i.e., they fulfilled minimum diagnostic criteria for PTSD. Those were enrolled in the 


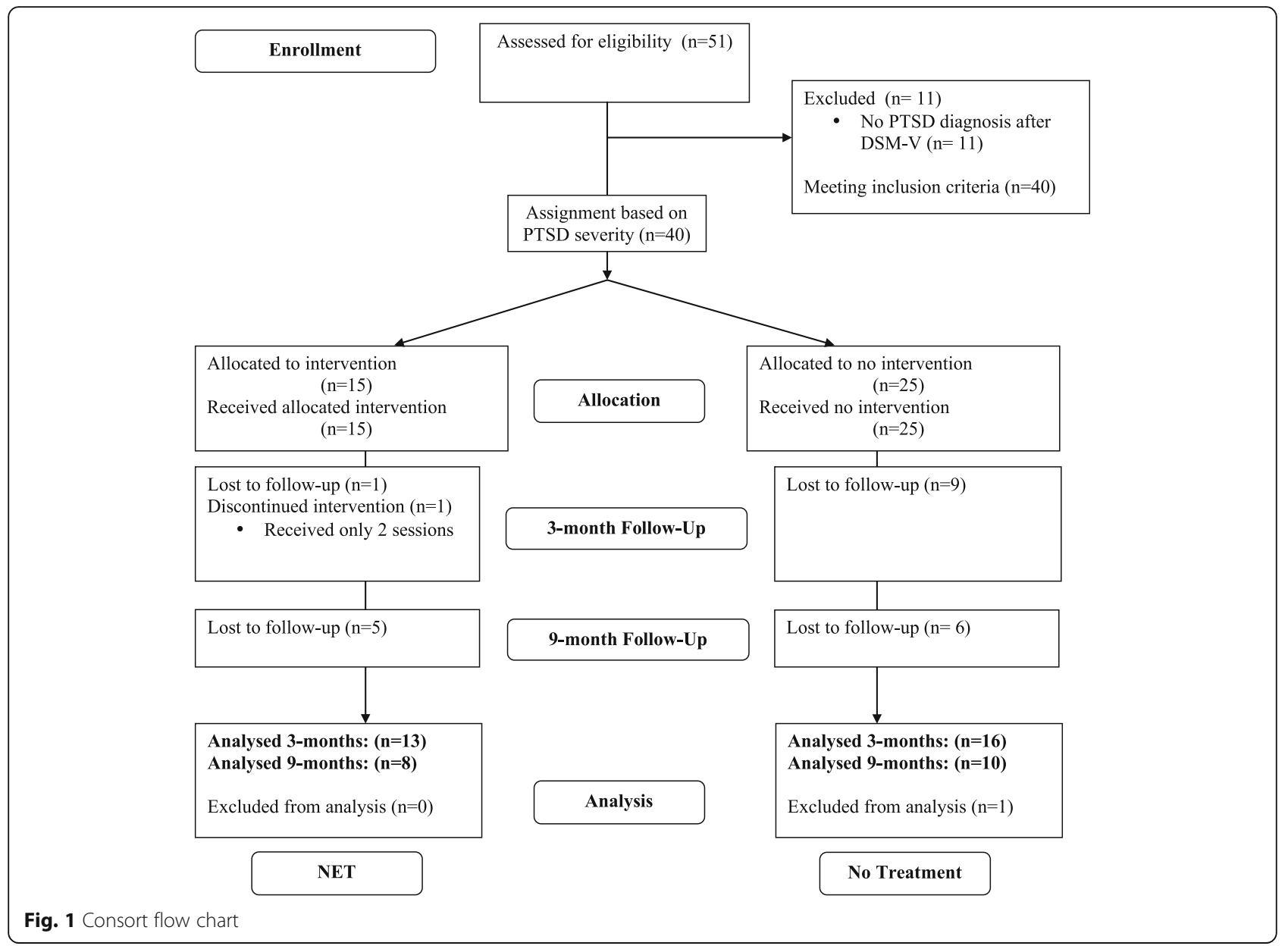

trial (Fig. 1). Exclusion criteria for receiving NET were pregnancy, ongoing substance abuse and obvious psychotic symptoms. None of these applied to the recruited participants. At baseline, 40 individuals were included, with 15 participants in the NET group and 25 individuals in the no treatment group. At 3-months follow-up, we relocated and assessed 29 participants with 13 participants in the NET group and 16 participants in the no treatment group. At 9-months follow-up, we relocated and assessed 18 participants, with 8 in the NET and 10 in the no treatment group. Participants who were lost or who dropped out were excluded from respective statistical analyses. While we focused mainly on adults we also included one minor, aged 14 years old.

Participants were informed verbally that their participation in the initial interview and potential intervention would be entirely voluntary and that they could withdraw from it at any time and for any reason without facing any negative consequences. They were informed about the objective of the assessment and the intervention, potential benefits and risks, confidentiality, and that no monetary compensation could be offered for the interview, or the time of treatment. Furthermore, the participants signed written informed consents, both in French and Kirundi, at the follow-up assessments consenting to their continued participation in the study and the anonymous use of the collected data for scientific purposes. They also consented in a separate written informed consent that parts of their anonymized narrations could be used for scientific purposes and trainings. The consents were read out and explained to participants who could not read. In the case of the underage participant, a guardian was asked to provide additional consent. No participant refused participation. For participating in the 9-months follow-up assessment the participants received a monetary compensation of 5000 BIF ( $3 €$ in April 2015) to offset any monetary costs (travel, loss of wages) associated with their participation in the study. The Ethical Review Board of the Université de Lumière of Bujumbura approved this study. The study was registered at Clinical Trials: UKCR2014.

\section{Setting, procedure, and research design}

This study was conducted between April 2014 and May 2015. The baseline assessment (baseline) started approximately 2 months after the flood disaster and 
was conducted in the emergency camps. The 3-months and 9-months follow-up assessments after the completion of NET were carried out in the participants' new homes, where they had resettled approximately 6 months following the disaster. The interviewers ensured privacy during the assessments by selecting locations where the conversation could not be overheard and that were as quiet as possible. Such locations included tents provided for that purpose in the emergency camps, and church facilities during the baseline assessments, and the homes of the participants during later follow-ups. The vast majority of the sample suffered from considerable mental health symptoms. Due to the humanitarian purpose of the project, ethical considerations and limited resources, we chose the 15 individuals most affected by PTSD symptoms to receive NET (NET group). The remaining participants did not receive an intervention (No Treatment group) but were assessed at the same time points as the NET group in order to monitor the development of their symptoms in the aftermath of the disaster. Individuals in the NET group received 6 sessions, once per week, with each session lasting between 1.5 and $2.5 \mathrm{~h}$ depending on the needs of the participant. One participant was excluded because she discontinued the NET after the second session due to personal reasons and continued it only after the 3-months follow-up. Another participant who had received NET could not be relocated for the 3-months follow-up. We lost 9 individuals of the No Treatment group at the 3-months follow-up because their place of residence could not be located. Due to political riots in Bujumbura in spring 2015 the 9-months follow-up assessment could not be completed. However, prior to stopping the project for security reasons, contact with eight participants of the NET group and 10 participants of the No Treatment group had resumed.

Twelve psychology students from the University Lumière of Bujumbura carried out the interviews under the supervision of the authors of this article. All of the students were in their final academic year of their undergraduate studies. The interviewers had been extensively trained in the relevant concepts of mental disorders, in using the employed psychometric instruments and had acquired supervised practical experience in previous research projects. Six of the students conducted the baseline assessment and assisted in organizing the follow-up assessments by maintaining contact with the participants. We aimed to keep the interviewers of the follow-up assessments blind to whether the participants received NET or did not receive any treatment. Hence six additional students joined the team, three at the 3-months and three at the 9-months follow-up assessment. To ensure high quality interviews and rigorous oversight, each interview was discussed afterwards with one of the authors.

\section{NET training and supervision}

The six students from the baseline-interview were selected to receive a six-day NET training by the first author in advance. The training emphasized the basic principals of NET and challenges in providing psychological assistance to people suffering from PTSD. Furthermore, the students were given time for practical exercises in groups to simulate sessions. After the successful completion of the training-course, each therapist received three clients. Five of the students carried out the NET interventions, the sixth student coordinated the activities and intervisions. Once a week, the therapies were supervised by the first author via skype. The trained students will be referred to as therapists in the remaining text.

\section{Instruments}

The following instruments have been used for the assessments at baseline, 3- and 9-months follow-ups. All instruments have been translated and blindly back translated by the research team and local translators from the English/French versions [48] into Kirundi. Difficulties or uncertainties appearing in the process were discussed in detail amongst German and Burundian mental health experts prior to data collection to ensure proper adaptation to the Burundian context. The majority of the instruments have been previously used in clinical research projects in Burundi $[49,50]$. WTP, stigmatization, and SES were only assessed at the follow-up assessments.

\section{Socio-economic status (SES)}

Participants were interviewed about their occupation prior to the flood and in the aftermath. Further, we assessed whether their earnings in the months prior to the follow-up assessments originated from donations, temporary work, or a more secure place of employment. The total amount of money available to the participants during each of these months was also recorded. In addition, the current living situation of each participant was assessed. They were asked whether they rented an apartment, owned property, lived on the streets, in a camp, or as a guest in another house and how much they paid or would have to pay for their accommodation. In addition, we asked how much money they had received from the Burundian Red Cross. We then calculated a mean value of the 3 months of income prior to the 3-months follow-up assessment.

\section{Traumatic events}

The number of traumatic event types experienced was assessed using a slightly adapted checklist that had 
already been used in a variety of contexts with populations affected by civil war, including Burundi [3, 50,51]. The list consisted of 29 items assessing the lifetime trauma events load. Seven items assessed specifically traumatic events and maltreatment (physical violence, sexual violence, emotional violence, neglect) during childhood. Events from the Posttraumatic Diagnostic Scale [52] were incorporated as well as different war-related witnessed and self-experienced events. Items were coded dichotomously with 0 (no) or 1 (yes) and summed up.

\section{PTSD symptom severity}

The PTSD Symptom Scale - Interview (PSS-I; [53]) is a semi-structured interview and was used to determine PTSD symptom severity. The instrument is validated to assess symptoms of PTSD experienced in the previous month and due to the most traumatic event that occurred at least 1 month before the assessment. The response is rated on a 4-point scale by the interviewer. Four items were added to adhere to the DSM-V classification of PTSD. The PSS-I has been tested in various cultural settings $[54,55]$ and showed good psychometric properties, e.g., inter-rater reliability $=0.93[53,56]$, and Cronbach's $\alpha=.90$; [55], including assessments conducted in Burundi (Cronbach's $\alpha=.94 ;$ [50]). The PSS-I has a maximum possible score of 60 .

\section{Depression symptom severity}

The Patient Health Questionnaire (PHQ-9; [57]) is a short, self-administered instrument which measures depression in adults. The PHQ consists of nine items which ask for the appearance of core symptoms of major depression disorder during the 2 weeks prior to assessment. The interviewer rates the severity of symptoms on a 4-point scale. The PHQ-9 has been previously tested in the Burundian context and showed good validity [58]. In the present study, PHQ-9 showed high internal consistency, as determined by Cronbach's $\alpha$ of .84 .

\section{Suicidal tendency}

We used Module $\mathrm{C}$ of version 6 of the Mini International Neuropsychiatric Interview (M.I.N.I.; [59]) to assess if participants reported a moderate or high risk of committing suicide. The M.I.N.I. is a short structured diagnostic interview for psychiatric disorders. It has been frequently used in a variety of cultures and settings [60].

\section{Willingness to Pay (WTP)}

Interviewers explained the concept of WTP to each participant. Further, the interviewers had been sensitized during their training to ensure that each participant understood that the responses they provided would not result in any negative consequence and that the amount of money attributed was only fictitious. The participants were then asked, "Knowing what you know of the beneficial effects of the therapeutic intervention regarding mental health symptoms today, what amount of money would you have been willing to reject in order to receive an intervention immediately." Hence, we used the concept WTP to assess how much money the participants would have rejected retrospectively. Taking the low education level of the participants into account the interviewers were trained to provide examples of what the participants might have afforded for the amount of money they reported being willing to refuse in order to ensure the participants had a meaningful understanding of the value they assigned to receiving the treatment. The amount of money was measured in Burundian franc (BIF) and later converted into $€$ with an exchange rate of 1661 BIF $\sim 1 €$.

\section{Stigmatization due to symptoms/treatment}

The Perception of Stigmatization by Others for Seeking Help (PSOSH; [61]) is a 5-item instrument to measure social and public stigma, as a result of seeking psychological help. The participants are asked how they expected people to react regarding their mental health symptoms, or when they would seek psychological treatment. Five possible reactions were offered including the following questions: "a)... react negatively towards you; b)... have a bad opinion about you; c)... perceive you as seriously imbalanced; d)... think of you in a less favourable way; e)... think that you represent a risk to another person?" Their responses were rated on a 5-point Likert-scale ranging from not at all (1) to a great deal (5). A higher perception of stigmatization is then translated into a higher sum score (5-25). The PSOSH has been tested in patients with PTSD and showed good validity and reliability (e.g. Cronbach's alpha $=.91$, test-retest reliability $=.82 ;[62,63])$. Of note is that the PSOSH has been developed for the field of school counselling. We adapted the original phrase for the purpose of this study to either assess stigmatization experienced or expected due to symptoms of PTSD or due to seeking treatment.

\section{Data analysis}

Data management and analysis were conducted using SPSS 20.0 [64] and R-Statistics [65]. There were no outliers and the data was normally distributed, as assessed by the Shapiro-Wilk test $(p>.05)$. All further assumptions were met, if not mentioned otherwise below. Repeated measure analysis (ANOVAs) were performed with time as a 3-level within-subject variable (baseline, 3 and 9-months follow-up) and treatment groups as 2-level between-subjects variable (NET group, No Treatment group). Due to the high dropout rate resulting from the political instability in spring 2015, we 
also conducted a repeated ANOVA including only the 3-months follow-up assessment to confirm the results. Because of the directional hypotheses regarding the effects of the intervention, and improvement of symptoms over time in the No Treatment group, analyses of interaction and time effects were computed one-tailed on an alpha level of .05 . The effect size for pairwise comparisons was estimated using Hedges' $g$ (Hedges, 1981). Hedges' $g$ was considered small with Hedges' $g \geq 0.20$, moderate with Hedges' $g \geq 0.50$, and large with Hedges' $g \geq 0.80$. To provide statistics for changes on the individual level, we calculated reliable change indices (RCIs; [66]) for changes in PTSD symptom severity and depression symptom severity. As a result of directional hypotheses, $R C I$-values greater than 1.65 indicated a significant difference.

As neither WTP nor SES were normally distributed (Shapiro-Wilk test: $p<.05$ ) and homogeneity of variances (Levene test: $p<.001$ ) was lacking amongst the treatment groups, we used Mann-Whitney $U$ and Wilcoxon-sign rank tests for comparisons. We tested whether the treatment groups differed in WTP and income at the 3-months follow-up. Furthermore, in the NET group we assessed if WTP changed between the follow-ups. Comparing stigmatization due to symptoms with stigmatization due to intervention at the 3-months follow-up, we conducted paired t-tests for each of the treatment groups. Furthermore, we assessed if the stigmatization due to symptoms reduced between the follow-ups in the NET group. Controlling for multiple comparisons we used the Bonferoni-Holm procedure. We then calculated a spearman-correlation to assess potential relationships between PTSD symptom severity, stigmatization due to symptoms, and stigmatization due to intervention.

\section{Results}

\section{Descriptive statistics at baseline}

The mean age of our sample was 28 years $(S D=11.7$ [14 to 78]). Approximately one third of the participants were illiterate $(n=11,37.9 \%)$. In total participants had received an average of 4 years of education $(M=3.8, S D=2.5)$. The average income across all participants for the first 3 months after successful completion of the therapy was $33.5 €(S D=$ 26.4) per month. There was no significant difference between treatment groups in any of the socio-demographic or socio-economic characteristics at baseline assessment, as assessed by $\chi^{2}$ and $\mathrm{t}$-tests (Tables 1 and 2).

As illustrated in Table 1 participants within the NET group suffered severely from PTSD symptoms $(M=35.9$, $S D=5.7)$ and from depression symptoms $(M=16.0, S D$ $=4.2$ ). Five participants reported moderate to severe suicidal tendencies at pretest. The No Treatment group was less affected than the NET group but still reported significant PTSD symptom severity $(M=20.3, S D=5.7)$ and depression symptom severity $(M=9.1, S D=3.2)$. Three
No Treatment group participants reported moderate to severe suicidal tendencies. Participants of both groups reported a similar amount of traumatic events experienced throughout lifetime.

\section{Mental health symptoms}

As the assumption of sphericity for the PTSD symptom severity was violated, we corrected the degrees of freedom using Greenhouse-Geisser estimates of sphericity. For the repeated measures ANOVA, we found a statistically significant time (baseline, 3-months, 9-months) $\mathrm{x}$ group (NET group, No Treatment group) interaction $\left(F(1.40,22.38)=4.29, p=.011, \eta_{\mathrm{p}}^{2}=.14\right)$. Furthermore, we found a significant main effect of time, $F(1.40,22.38)$ $=38.47, p<.001, \eta_{\mathrm{p}}^{2}=.60$ ), and a non-significant main effect of group, $\left.F(0.70,11.19)=2.06, p=.17, \eta_{\mathrm{p}}^{2}=.05\right)$. Overall, the PTSD symptoms severity improved significantly in both groups from baseline to 3-months follow-up (Hedges' $g_{N E T}=1.62 ;$ Hedges' $g_{N o}$ Treatment $=0.64)$ and from baseline to 9-months follow-up (Hedges' $g_{N E T}=3.44$; Hedges' $\left.g_{\text {No Treatment }}=2.55\right)$. Overall, the improvements were more pronounced in the NET group. On the individual level the RCIs indicated that a high percentage of participants in the NET group suffered significantly less from PTSD symptoms (75\%) or reported an unchanged symptom severity $(25 \%)$ at 3 -months follow-up, while $100 \%$ of them had significantly improved at 9-months follow-up. The majority of the participants of the No Treatment group suffered less (60\%), while $30 \%$ remained unchanged, and $10 \%$ deteriorated regarding symptom severity at 3-months follow-up. At 9-months follow-up, $80 \%$ of the No Treatment group had improved significantly, and $20 \%$ had remained unchanged.

Further, we calculated a second repeated measures ANOVA for the 3-months follow-up to account for the participant attrition due to the political instability in Burundi. We found a significant interaction of time (baseline, 3-months) x group (NET group, No Treatment group; $\left.F(1,27)=4.07, p=.027, \eta_{p}^{2}=.07\right)$, a significant main effect of time $\left(F(1,27)=20.68, p<.001 ; \eta_{p}^{2}=.27\right)$, and a significant main effect of group $(F(1,27)=38.25$, $p<.001 ; \eta_{p}^{2}=.43$ ) (Fig. 2).

In the repeated measures ANOVA regarding depression symptom severity, we found a statistically significant time (baseline, 3-months, 9-months) $\mathrm{x}$ group (NET group, No Treatment group) interaction $(F(2,32)=4.59$, $\left.p=.009, \eta_{p}^{2}=.13\right)$. Furthermore, we found a significant main effect of time, $\left(F(2,32)=9.35, p<.001, \eta_{p}^{2}=.23\right)$, and a significant main effect of group $(F(1,16)=8.26, p$ $\left.=.011, \eta_{p}^{2}=.20\right)$. Overall, the depression symptom severity improved significantly in the NET group from baseline to 3-months follow-up (Hedges' $g_{N E T}=0.84$; Hedges' $\left.g_{\text {No Treatment }}=-0.06\right)$ and from baseline to 9-months 
Table 1 Socio-demographic data and outcome assessments at pretest

\begin{tabular}{|c|c|c|c|c|c|c|c|c|c|c|c|c|c|c|c|c|c|}
\hline & & \multicolumn{4}{|c|}{ No Treatment } & \multicolumn{4}{|c|}{ Narrative Exposure Therapy } & \multicolumn{4}{|c|}{ Total } & \multirow[b]{2}{*}{$\overline{x^{2}}$} & \multirow[b]{2}{*}{$p$} & \multirow[b]{2}{*}{$T$} & \multirow[b]{2}{*}{$p$} \\
\hline & & $\bar{M}$ & SD & $n$ & $\%$ & $\bar{M}$ & SD & $n$ & $\%$ & $\bar{M}$ & SD & $n$ & $\%$ & & & & \\
\hline Age in years & & 29.8 & 14.9 & & & 26.3 & 5.8 & & & 28.2 & 11.7 & & & & & 0.87 & .40 \\
\hline \multirow[t]{2}{*}{ Sex } & Male & & & 2 & 12.5 & & & 3 & 23.0 & & & 5 & 17.2 & 0.07 & .80 & & \\
\hline & Female & & & 14 & 87.5 & & & 10 & 77.0 & & & 24 & 82.7 & & & & \\
\hline \multirow[t]{2}{*}{ Illiteracy } & No & & & 10 & 62.5 & & & 8 & 61.5 & & & 18 & 62.1 & 0.00 & .99 & & \\
\hline & Yes & & & 6 & 37.5 & & & 5 & 38.5 & & & 11 & 37.9 & & & & \\
\hline Education in years & & 3.9 & 2.1 & & & 3.7 & 2.9 & & & 3.8 & 2.5 & & & & & 0.25 & .80 \\
\hline \multirow[t]{5}{*}{ Marital status } & Single & & & 3 & 18.8 & & & 3 & 23.0 & & & 6 & 20.7 & 4.65 & .33 & & \\
\hline & Married & & & 6 & 37.5 & & & 2 & 15.4 & & & 8 & 27.6 & & & & \\
\hline & Divorced & & & 1 & 6.3 & & & 0 & 0.0 & & & 1 & 3.5 & & & & \\
\hline & Widowed & & & 1 & 6.3 & & & 4 & 30.8 & & & 5 & 17.2 & & & & \\
\hline & In relationship & & & 5 & 31.3 & & & 4 & 30.8 & & & 9 & 31.0 & & & & \\
\hline \multirow[t]{5}{*}{ Number of children lost } & 0 & & & 8 & 50.0 & & & 3 & 23.1 & & & 11 & 37.9 & 3.78 & .44 & & \\
\hline & 1 & & & 4 & 25.0 & & & 5 & 38.5 & & & 9 & 31.0 & & & & \\
\hline & 2 & & & 2 & 12.5 & & & 4 & 30.8 & & & 6 & 20.7 & & & & \\
\hline & 3 & & & 1 & 6.3 & & & 1 & 7.6 & & & 2 & 6.9 & & & & \\
\hline & 4 & & & 1 & 6.3 & & & 0 & 0.0 & & & 1 & 3.5 & & & & \\
\hline \multirow[t]{3}{*}{ Occupation before flood disaster } & None & & & 2 & 12.5 & & & 0 & 0.0 & & & 2 & 6.9 & 3.12 & .54 & & \\
\hline & Student & & & 1 & 6.3 & & & 0 & 0.0 & & & 1 & 3.5 & & & & \\
\hline & Some job & & & 13 & 81.3 & & & 13 & 100.0 & & & 27 & 89.6 & & & & \\
\hline \multirow[t]{3}{*}{ Occupation after flood disaster } & None & & & 1 & 6.3 & & & 2 & 15.4 & & & 3 & 10.3 & 2.25 & 69 & & \\
\hline & Student & & & 1 & 6.3 & & & 0 & 0.0 & & & 1 & 3.5 & & & & \\
\hline & Some job & & & 14 & 87.5 & & & 11 & 84.6 & & & 25 & 86.2 & & & & \\
\hline Traumatic events & & 16.9 & 5.4 & 16 & & 14.0 & 4.4 & 13 & & 18.0 & 4.9 & 29 & & & & 1.43 & .17 \\
\hline Depression symptom severity ${ }^{* * *}$ & & 9.1 & 3.2 & 16 & & 16.0 & 4.2 & 13 & & 12.2 & 5.1 & 29 & & & & 4.88 & $<.001$ \\
\hline PTSD symptom severity ${ }^{* * *}$ & & 20.3 & 5.7 & 16 & & 35.9 & 5.7 & 13 & & 27.3 & 9.7 & 29 & & & & 7.29 & $<.001$ \\
\hline
\end{tabular}

***indicate significant differences with $p \leq .001$

follow-up in both groups (Hedges' $g_{N E T}=1.88$; Hedges' $g_{\text {No }}$ Treatment $=0.72$ ). Overall, the improvements were more pronounced in the NET group. On the individual level the RCIs indicated that a high percentage of participants of the NET group suffered significantly less from depression symptoms (50\%), while some reported an unchanged symptom severity (37.5\%), and a minority reported deteriorated symptom severity (12.5\%) at 3-montsh follow-up, while 100\% of them had improved significantly at 9-months follow-up. Approximately one-third of the participants of the No Treatment group suffered less $(30 \%)$ from depression symptoms, while

Table 2 Socio-economic data

\begin{tabular}{|c|c|c|c|c|c|c|c|c|c|c|c|c|c|c|c|c|}
\hline & & \multicolumn{4}{|c|}{ No Treatment } & \multicolumn{4}{|c|}{$\begin{array}{l}\text { Narrative Exposure } \\
\text { Therapy }\end{array}$} & \multicolumn{4}{|c|}{ Total } & \multirow[b]{2}{*}{$\overline{x^{2}}$} & \multirow[b]{2}{*}{$T$} & \multirow[b]{2}{*}{$p$} \\
\hline & & $\bar{M}$ & SD & $n$ & $\%$ & $\bar{M}$ & SD & $n$ & $\%$ & $\bar{M}$ & SD & $n$ & $\%$ & & & \\
\hline \multicolumn{2}{|c|}{$\begin{array}{l}\text { Average income per month in the first } 3 \text { months after treatment } \\
\text { completion }(€)\end{array}$} & 31.5 & 22.9 & & & 36.0 & 31.0 & & & 33.5 & 26.4 & & & & 0.44 & .67 \\
\hline \multicolumn{2}{|l|}{ Costs housing 3-months follow-up (€) } & 12.6 & 6.2 & & & 14.8 & 5.8 & & & 13.3 & 6.0 & & & & 0.72 & .49 \\
\hline \multirow[t]{5}{*}{ Housing condition 3-months follow-up } & Rent & & & 12 & 75.0 & & & 7 & 53.8 & & & 19 & 65.5 & 0.64 & & .42 \\
\hline & Property & & & 0 & 0.0 & & & 0 & 0.0 & & & 0 & 0.0 & & & \\
\hline & Guest & & & 4 & 25.0 & & & 6 & 46.2 & & & 10 & 34.5 & & & \\
\hline & On the street & & & 0 & 0.0 & & & 0 & 0.0 & & & 0 & 0.0 & & & \\
\hline & In a camp & & & 0 & 0.0 & & & 0 & 0.0 & & & 0 & 0.0 & & & \\
\hline
\end{tabular}



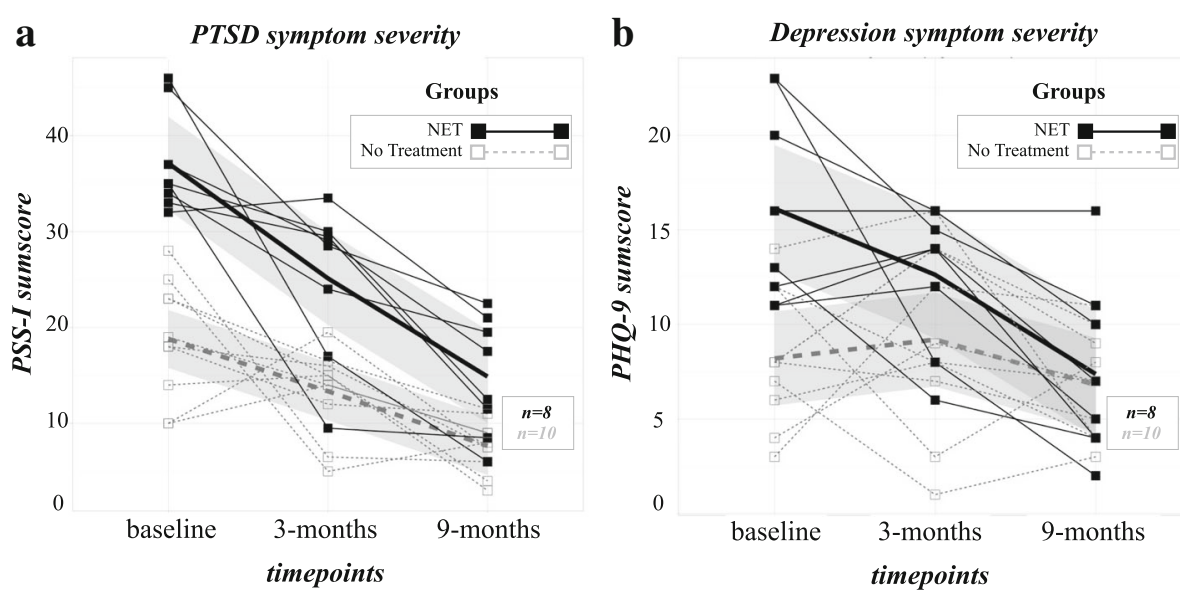

Fig. 2 Spaghetti plots of (a) PTSD symptom severity and (b) Depression symptom severity at baseline, 3-months and 9-months follow-up for the individuals in the Narrative Exposure Therapy group (in black) or the No Treatment group (in dashed grey) respectively. Thicker lines represent the linear model, Grey shaded areas represent the standard error

another third remained unchanged (30\%), and the majority $(40 \%)$ deteriorated regarding symptom severity at 3-months follow-up. At 9-months follow-up, the depression symptom severity of $50 \%$ of the No Treatment group had improved significantly, while the symptoms of $30 \%$ had remained unchanged, and of $20 \%$ had deteriorated significantly.

Further, we calculated a second repeated measures ANOVA for the 3-months follow-up to encounter the drop-out due to the political instability in Burundi. We found a significant interaction of time (baseline, 3-months) $\mathrm{x}$ group (NET group, No Treatment group; $F(1,27)=3.57$, $\left.p=.035, \eta_{p}^{2}=.06\right)$, a non-significant main effect for time $\left(F(1,27)=2.71, p<.055, \eta_{p}^{2}=.04\right)$, and a significant main effect for group, $\left(F(1,27)=20.28, p<.001, \eta_{p}^{2}=.429\right)$.

\section{WTP and monthly income}

Assessing WTP and monthly income, we excluded one participant in the NET group at 3-months follow-up because of missing data. In total we included 28 participants $\left(n_{N E T}=12 ; n_{N o}\right.$ Treatment $\left.=16\right)$ for the 3 -months and 18 participants $\left(n_{N E T}=7 ; n_{N o}\right.$ Treatment $\left.=10\right)$ for the 9-months follow-up.

As illustrated in Fig. 3, WTP was significantly higher than the monthly income for participants in the NET group at 3-months follow-up ( $W=73.0, z=-2.85, p$ $<.005)$. In addition, WTP in the NET group was significantly higher at 9-months compared to 3-months follow-up ( $W=28.0, z=-2.418, p=.016)$. Compared with the No Treatment group, the NET group reported a significantly higher WTP at 3-months follow-up $(U=22.5, z=-$ 3.504, $p<.001)$. Still, the No Treatment group reported a WTP comparable to their monthly income to receive the intervention immediately ( $W=68.0, z=0.0, p=1.0)$.

\section{Stigmatization due to symptoms of PTSD \& seeking treatment}

As illustrated in Fig. 4, we found a non-significant mean difference of stigmatization due to symptoms and stigmatization due to treatment in the NET group at 3 -months follow-up $(t(12)=2.08, p=.0595$, Hedges' $g=$ 0.62). However, a trend toward significance could be observed with a medium effect size: Participants of the NET group reported more stigmatization due to symptoms $(M=14.77, S D=5.83)$ than stigmatization due to treatment $(M=11.69, S D=3.57)$. The stigmatization due to symptoms reduced between the 3-months $(M=15.38$, $S D=6.39)$ and the 9-months follow-up $(M=11.00, S D=$ $5.40)$ within the NET group $(t(7)=4.162, p=.004$, Hedges' $g=0.70)$. The No Treatment group did not differ regarding stigmatization due to symptoms $(M=9.44, S D=3.56)$ and expected stigmatization due to treatment $(M=8.33, S D=$ 3.20) at 3-months follow-up $(t(15)=0.98, p=.34$, Hedges' $g=0.32$ ). Within the NET group, PTSD symptoms severity correlated significantly with stigmatization due to symptoms at 3-months $(r(11)=.55, p=.051)$ and 9-months $(r(6)=.71, p=.048)$ follow-up and did not correlate significantly with stigmatization due to intervention at 3-months $(r(11)=0.21, p=.49)$ or 9-months follow-up $(r(6)=0.57, p=.14)$.

\section{Discussion}

The present paper provided evidence for the feasibility of conducting an evidence based, trauma-focused exposure therapy such as NET with survivors of natural disasters in a post-conflict region while they are still early in the recovery process. Symptom severity of PTSD (Hedges' $g_{N E T}=3.44$; Hedges' $g_{N o}$ Treatment $\left.=2.55\right)$ and depression $\left(\right.$ Hedges' $g_{N E T}=1.88 ;$ Hedges' $\left.g_{\text {No Treatment }}=0.72\right)$ improved significantly in both groups at 9-months follow-up with a 

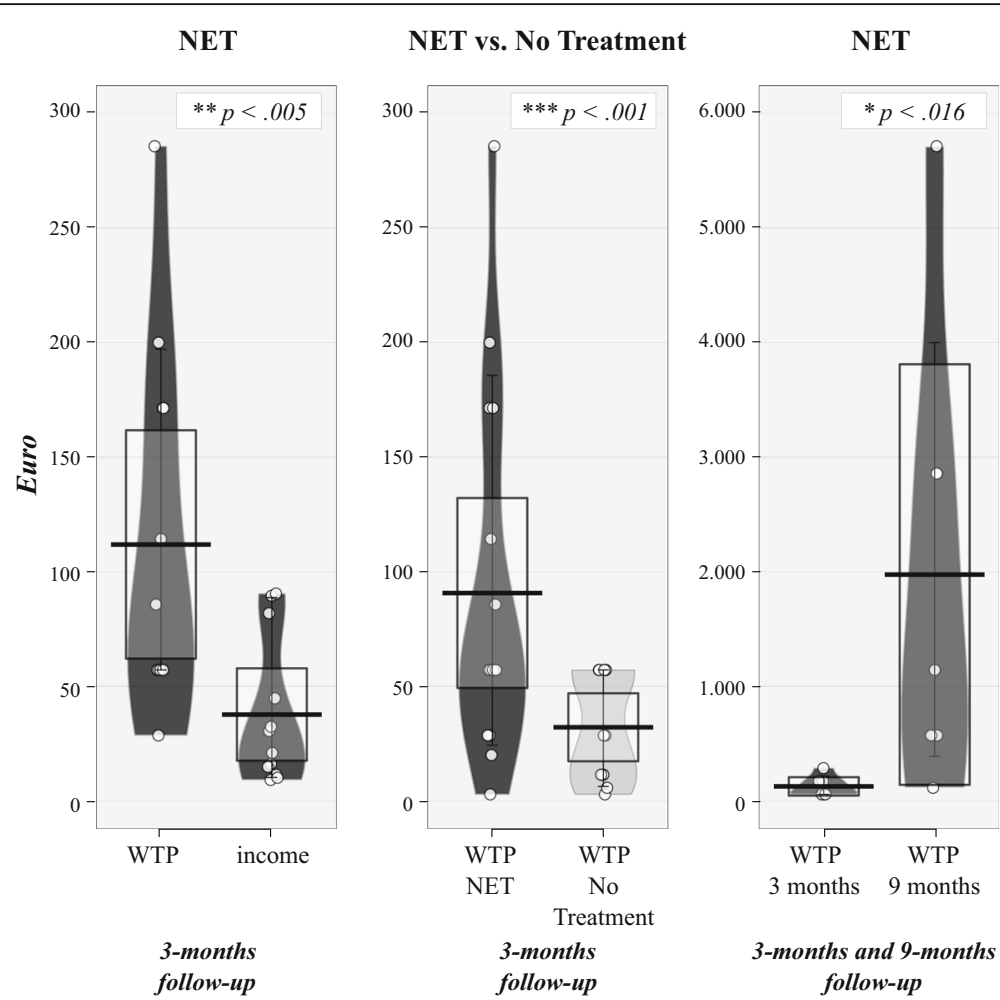

Fig. 3 RDI (Raw data, Descriptive and Inferential statistics) plots of average income and Willingness To Pay (WTP) at 3-months and 9-months follow-up for the individuals in the Narrative Exposure Therapy group or the No Treatment group respectively. Dots represent the raw data, vertical black bar shows central tendency, bean representing a smoothed density, whisker representing 95 confidence interval

greater improvement amongst those participants who had received NET. In addition, participants in the NET group assigned great value to the psychological intervention by indicating their willingness to refuse a sum equal to 1 month's income (35 €) at 3-months and a sum equal to more than a year of income at 9-months follow-up to receive such an intervention immediately in the aftermath of the disaster. Finally, we found a significant decrease of stigmatization due to symptoms of PTSD in the NET group between the 3-months and the 9-months follow-up, indicating that addressing mental health issues using evidence-based treatment most likely improves the well-being of individuals by reducing or even removing daily stressors such as stigmatization.

In the aftermath of the flood disaster, we relied on volunteers of the Burundian Red Cross to identify survivors they considered most mentally affected according to the following three criteria: (1) having lost a close relative; (2) being isolated or behaving strangely in the camp; and (3) hearing about symptoms such as nightmares, sleeping troubles, or angry outbursts. Following our screening criteria, 40 out of 51 survivors we enrolled fulfilled enough symptoms to diagnose them with PTSD. This result suggests that identifying affected survivors is feasible in emergency camps and can be carried out by briefed volunteers. Furthermore, it might reflect a generally high prevalence of trauma-related mental health disorders in such regions.

Survivors in the NET group suffered from severe symptoms of PTSD and depression and often experienced multiple traumatic experiences besides the natural disaster, including events related to the civil war. We were able to replicate the beneficial effect of NET regarding mental health symptoms in the aftermath of a natural disaster [23, 24]. As in many other studies (for an overview see [25]), we found the strongest improvement of mental health symptoms after a longer period of time had passed. This finding is usually attributed to the idea that during NET a memory process is started which continues over the following months and years and causes an ongoing improvement of symptoms. This process is related to dissolving the fear network through neural changes due to elaboration and overcoming avoidance behaviour. The sustainable effect of NET has been well-established over the past 15 years and is considered a particular strength of this approach [30, 32].

The beneficial effect of NET within this sample was found even though both follow-up assessments were conducted at the respective beginning of the two rainy seasons in Burundi. Particularly, the rain falling during 


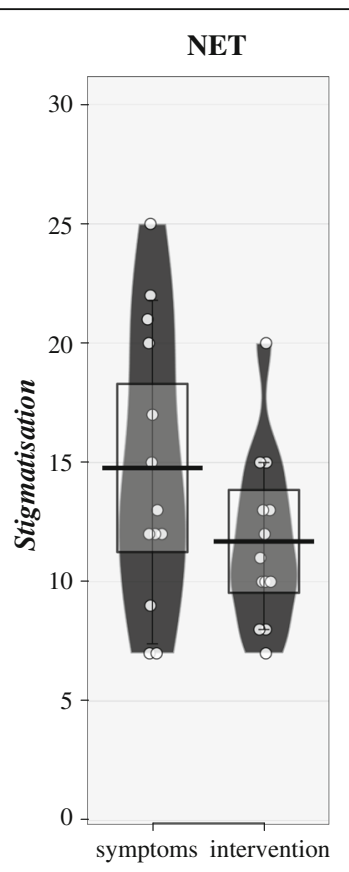

3-months follow-up

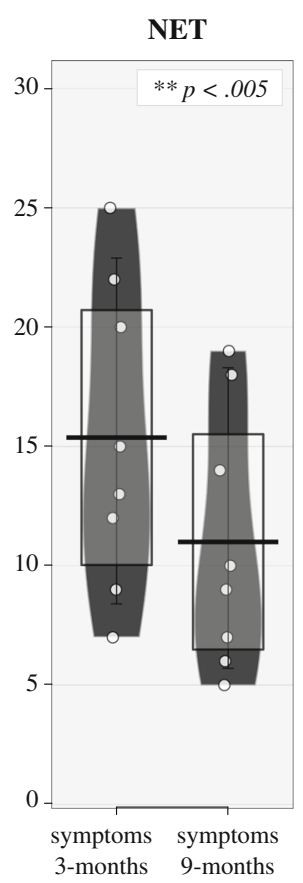

3-months and 9-months follow-up

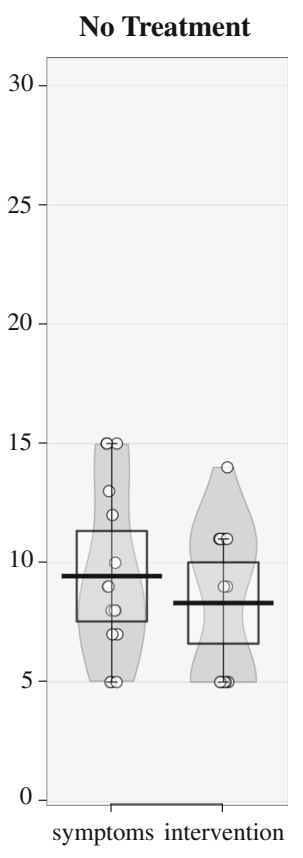

3-months follow-up

Fig. 4 RDI (Raw data, Descriptive and Inferential statistics) plots of average stigmatization concerning symptoms and intervention at 3-months and 9-months follow-up for the individuals in the Narrative Exposure Therapy group or the No Treatment group respectively. Dots represent the raw data, vertical black bar shows central tendency, bean representing a smoothed density, whisker representing 95 confidence interval

3-months follow-up assessment triggered many memories and elicited past fears among the participants. After the closure of the emergency camps, the majority of participants moved back to the same suburb of Bujumbura where they had resided prior to the flood disaster. Many survivors were therefore exposed to locations and cues related to the loss of their loved ones. Furthermore, many of the participants struggled with their grief associated with the death of their children. Shortly prior to our first follow-up assessment, the Burundian government had conducted a memorial service for all families who had lost their children during the flood disaster. Unfortunately, two of the participants within the NET group reported that they had not been officially recognized as parents who had lost their children, which caused increased mental health symptoms and even suicidal ideations. Due to ethical considerations we offered some support monitoring their suicidal ideations and conducted a memorial with those two participants to acknowledge their losses thereby assisting them to overcome those ideations.

The PTSD and depression symptoms of participants less affected from trauma-related mental health disorders in the aftermath of the natural disaster also improved significantly over time. This result indicates that less severely affected individuals might not require a trauma-specific intervention but might benefit from spontaneous remission when they slowly regain the previous standard of living. Overall, the mental health improvements of both groups, the no treatment and the intervention group, are in line with the assumptions that more severely affected individuals with an elevated risk of chronicity of PTSD symptoms [67], might benefit from trauma-focused interventions while less affected individuals might recover spontaneously. However, due to limitations of the study's design we can not rule out the possibility that the less affected individuals might also have profited from NET, nor that the more severely affected individuals might have recovered spontaneously.

As in previous studies $[26,28]$, we demonstrated that the dissemination of NET to relatively inexperienced local counsellors is feasible within a short training period and yields very promising results regarding mental health benefits. In addition, we argue that the use of local counsellors counteracts stigmatization and supports the aim of NET of being aware of local traditions and cultural characteristics. These factors along with the fact that local counsellors advocated the intervention might have improved the acceptance amongst the beneficiaries. 
At 3-months follow-up, participants in both groups were willing to retrospectively refuse a sum equal to at least the income of 1 month in order to receive an individual trauma-focused intervention while still living in the emergency camps. In the NET group, the amount was significantly higher and increased over time.This result emphasizes the argument made by Schauer and Schauer [15] that providing "appropriate mental health services for trauma victims ... [is] anything but a "luxury" especially in resource poor, conflict ridden countries ([16], 4-5, pp.)." Furthermore, the quantitative results are in line with various qualitative accounts of traumatized survivors across different cultures whose testimonies provide evidence that narrating and acknowledging their stories through the process of NET is highly appreciated ([16, 68, 69], 4-5, pp.). We can only speculate if the participants' high WTP is mainly related to improved mental health [25] including overcoming survivor's guilt, shame, and feelings of revenge $[11,70]$, or improved status due to improved functioning $[6,71]$, or both. Nevertheless, the amounts reported indicate that this population is aware of the impairment they suffer due to mental health problems and that they are assigning a significant value to receiving treatment. The fact that the relative WTP of our sample (100\% of monthly income) was much higher than the WTP of a sample of depressed American patients (9\% of monthly income) [37] might be attributed to differences of the samples regarding general income and living conditions, culture, diagnostics, and to the fact that we asked how much money our participants would have refused in order to receive treatment as soon as possible instead of asking them to pay that amount. However, it might also emphasize how much appropriate mental health treatments are valued in post-conflict countries.

Stigmatization and resentment due to competition regarding scarce resources present a serious obstacle to psychological interventions in emergency camps. Even more so, as there is almost no privacy in such circumstances and others notice when someone receives extra attention in the form of a therapeutic intervention in the camps. In light of this, we provided psychoeducation to the community in order to counter this obstacle to participation in the treatment. Over the course of the intervention, all but one participant completed the NET while living in the emergency camps. Furthermore, participants of both groups indicated at the first follow-up that they did not or did not expect to feel more stigmatized due to receiving trauma-focused treatment than they felt stigmatized due to their symptoms. The NET group even reported that they felt less stigmatized by the fact that they had received treatment than because of their symptoms. The continuous association between PTSD symptoms and feeling stigmatized indicates that stigmatization due to symptoms most likely severely affects the social status of individuals in their community and hence elicit ongoing suffering. Overall, these results strongly contradict the idea that proactively addressing mental health problems in severely affected populations living in circumstances that offer little privacy would aggravate the situation of affected individuals due to stigmatization. Instead, addressing mental health issues using evidence-based treatment most likely improves the well-being of individuals by removing daily stressors in the form of stigmatization [14].

The presented study has the following caveats. The non-randomized design, the small sample size, the loss of participants during the follow-ups, and the possible regression to the mean are confounding factors. It is possible that the within group pre-post differences and the between group differences of the mental health symptoms arise from those factors. Hence the conclusions regarding the development of mental health symptoms have to be considered with a grain of salt. According to a post-hoc sample size calculation, assuming Bonferoni corrected significance for testing PTSD and depression levels, and a power of .8 , future studies aiming to replicate the mental health related effects of NET in the aftermath of a natural disaster would require a total sample size of at least 16 participants. Regarding the feasibility of dissemination of the NET approach to local counsellors in short trainings, we must acknowledge that a 6-day training is unusually short. Such a short training was only feasible because the therapists already had extensive experience in mental health diagnostics, and had some background knowledge and experience due to their study of psychology in Burundi. Moreover, we would like to stress the necessity of supervision for newly trained therapists as they are quickly confronted with very severe cases including dissociation and suicidal ideations.

It is important that we address our attempt to operationalize cultural acceptance via the concept of WTP. We are aware that the concept of assigning a monetary value to assess the subjectively-perceived benefits of an intervention itself might be a new and maybe even capitalistic idea. Furthermore, this approach is arguably reducing the benefits of a therapy to a single dimension. As the amounts of money were fictional we cannot rule out that the participants exaggerated the amount they would have been willing to refuse in retrospect.

\section{Conclusions}

Implementing such a project in emergency camps about 2 months after the disaster required us to consider the following obstacles: (1) Potential stigmatization and resentment because of the extra attention some of the survivors received due to our intervention; (2) rumours and worries including ideas about witchcraft we might use when talking 
in private with the participants; and (3) frustration and confusion about psychological interventions in general. According to accounts of Red Cross volunteers and of survivors in the emergency camps, the psychological response of institutions and NGOs had not been well-coordinated within the emergency camps. The different approaches, lack of transparency and psychoeducation had confused the survivors in the camps and resulted in mistrust towards psychologists.

While navigating these challenges, we demonstrated that implementing specialized trauma-focused treatment in the aftermath of disaster is feasible in post-conflict regions. NET has been confirmed as a promising approach for severely affected individuals given that it can be disseminated to lay counsellors, both directly and via train-the-trainer models [28]. At the same time our experiences during the implementation of the project indicate the need for (1) reinforcing and expanding coordinated mental health approaches for interventions in the aftermath of disasters in order to to combine activities of NGOs instead of diffusing impact due to confusion created by different approaches without coordination; (2) evaluating interventions in this context; (3) implementing evidence-based trauma-focused interventions while also addressing grief and ideas of revenge that arose in some survivors as they tried to deflect blame and attribute meaning to their losses; and (4) building up mental health capacities in post-conflict countries such as Burundi. The results indicated furthermore that NET is well received by the affected populations and that NET might contribute significantly to reducing daily stressors associated with stigmatization due to PTSD.

\section{Abbreviations}

NET: Narrative Exposure Therapy; PTSD: Posttraumatic stress disorder;

SES: Socio economic status; WTP: Willingness to pay

\section{Acknowledgements}

We would like to thank Hervé Mugisha, Jean-Arnaud Muhoza, Thierny Ndajikengurukiye, Lydia Nitanga, Amini Ahmed Rushoza, and Anitha Ziser for implementing treatments and initial assessments, and assisting with the coordination of the project in Burundi. We are grateful to Gina Gatore, Alice Kanyambo, Adéline Mubona, Gynelle Mugisha, Jean Claude Ninziza, and Cynthia Nishinwe for conducting the follow-up interviews, to Jean-Baptiste Niyungeko, the coordinator of emergency response with the Burundian Red Cross for initializing the cooperation, to Justin Preston for reviewing the manuscript prior to submission, and to Dr. Manassé Bambonyé for his guidance during the implementation of the project. Furthermore, we thank the Burundian Red Cross and the Burundian Red Cross volunteers for their support and logistic assistance.

\section{Funding}

The funding for this project was provided by the Non-Governmental Organization vivo international e.V. (vivo). Vivo is committed to the evaluation and dissemination of evidence-based psychological intervention with a particular focus on trauma-related disorders (for more details see https://www.vivo.org/). SS was funded by the Deutscher Akademischer Austauschdienst.

\section{Availability of data and materials}

The datasets used and/or analyzed during the current study are available from the corresponding author on reasonable request.

\section{Authors' contributions}

The study was designed by AC and SS. Both supervised the assessments, which were conducted by local psychology students. AC coordinated the cooperation of the project partners, organized the funding, trained the local psychology students and supervised the treatments. SS coordinated the follow-up assessments, analyzed the data, and wrote the initial draft under the guidance of AC. All authors revised the manuscript and approved the final version.

\section{Ethics approval and consent to participate}

The research project was approved by the ethics committee of the University Lumière of Bujumbura, Burundi. All participants provided written informed consent to participate in this study. In the case of minor-aged participants, a guardian provided additional written informed consent.

\section{Consent for publication}

Not applicable

\section{Competing interests}

The authors declare that they have no competing interests.

\section{Publisher's Note}

Springer Nature remains neutral with regard to jurisdictional claims in published maps and institutional affiliations.

\section{Author details}

${ }^{1}$ Department of Psychology, University of Konstanz, Konstanz, Germany. Department of Psychology, University Lumière of Bujumbura, Bujumbura, Burundi. ${ }^{3}$ Non-Governmental Organization Psychologues sans Frontières Burundi, Bujumbura, Burundi. ${ }^{4}$ Non-Governmental Organization vivo international e.V., Konstanz, Germany. ${ }^{5}$ Department of Cognitive and Clinical Neuroscience, Central Institute of Mental Health, Medical Faculty Mannheim, University of Heidelberg, Mannheim, Germany. ${ }^{6}$ Graduate School of Economic and Social Sciences, University of Mannheim, Mannheim, Germany.

Received: 18 October 2017 Accepted: 25 June 2018

Published online: 18 July 2018

\section{References}

1. Gulliver SB, et al. The psychological consequences of disaster. In: Ouimette P, Read JP, editors. Trauma and substance abuse: causes, consequences, and treatment of comorbid disorders. Washington DC: American Psychological Association; 2014. p. 125-41.

2. Galea S, Nandi A, Vlahov D. The epidemiology of post-traumatic stress disorder after disasters. Epidemiol Rev. 2005;27:78-91.

3. Neuner F, et al. Psychological trauma and evidence for enhanced vulnerability for posttraumatic stress disorder through previous trauma among West Nile refugees. BMC Psychiatry. 2004;4:34.

4. Wilker $S$, et al. How to quantify exposure to traumatic stress? Reliability and predictive validity of measures for cumulative trauma exposure in a postconflict population. Eur J Psychotraumatol. 2015;6:28306.

5. Neuner F, et al. Post-tsunami stress: a study of posttraumatic stress disorder in children living in three severely affected regions in Sri Lanka. J Trauma Stress. 2006;19(3):339-47.

6. Chisholm D, et al. Scaling-up treatment of depression and anxiety: a global return on investment analysis. Lancet Psychiatry. 2016;3(5):415-24.

7. Weiss MG, et al. Mental health in the aftermath of disasters: consensus and controversy. J Nerv Ment Dis. 2003;191(9):611-5.

8. Fernando GA, Miller KE, Berger DE. Growing pains: the impact of disasterrelated and daily stressors on the psychological and psychosocial functioning of youth in Sri Lanka. Child Dev. 2010;81(4):1192-210.

9. Miller KE, Rasmussen A. War exposure, daily stressors, and mental health in conflict and post-conflict settings: bridging the divide between traumafocused and psychosocial frameworks. Soc Sci Med. 2010;70(1):7-16.

10. McFarlane AC. Helping the victims of disasters. In: Freedy JR, Hobfall SE, editors. Traumatic stress: from theory to practice. New York: Plenum Press; 1995. p. 287-314.

11. Bayer CP, Klasen F, Adam H. Association of trauma and PTSD symptoms with openness to reconciliation and feelings of revenge among former Ugandan and Congolese child soldiers. J Am Med Assoc. 2007;298(5):555-9.

12. Mathews A, MacLeod C. Cognitive vulnerability to emotional disorders. Annu Rev Clin Psychol. 2005:1:167-95.

13. Elbert T, Schauer M. Burnt into memory. Nature. 2002;419(6910):883. 
14. Neuner F. Assisting war-torn populations: should we prioritize reducing daily stressors to improve mental health? Comment on Miller and Rasmussen (2010). Soc Sci Med. 2010;71:1381-4.

15. Schauer M, Schauer E. Trauma-focused public mental-health interventions: a paradigm shift in humanitarian assistance and aid work. In: Martz E, editor. Trauma Rehabilitation After War and Conflict. New York: Springer Science +Business Media; 2010

16. Schauer M, Neuner F, Elbert T. Narrative Exposure Therapy: a short-term treatment for traumatic stress disorders. Toronto: Hogrefe \& Huber; 2011

17. Cienfuegos AJ, Monelli C. The testimony of political repression as a therapeutic instrument. Am J Orthop. 1983;53(1):43-51.

18. Foa EB, Rothbaum BO. Treating the trauma of rape: cognitive-behavioral therapy for PTSD. New York: Guilford Press; 2001.

19. Hensel-Dittmann D, et al. Treatment of traumatized victims of war and torture: a randomized controlled comparison of Narrative Exposure Therapy and stress inoculation training. Psychother Psychosom. 2011;80(6):345-52.

20. Neuner F, et al. Can asylum-seekers with posttraumatic stress disorder be successfully treated? A randomized controlled pilot study. Cogn Behav Ther 2010;39(2):81-91.

21. Schaal S, Elbert T, Neuner F. Narrative Exposure Therapy versus interpersonal psychotherapy. A pilot randomized controlled trial with Rwandan genocide orphans. Psychother Psychosom. 2009;78(5):298-306.

22. Stenmark H, et al. Treating PTSD in refugees and asylum seekers within the general health care system. A randomized controlled multicenter study. Behav Res Ther. 2013;51:641-7.

23. Catani $\mathrm{C}$, et al. Treating children traumatized by war and tsunami: a comparison between exposure therapy and meditation-relaxation in NorthEast Sri Lanka. BMC Psychiatry. 2009;9:22.

24. Zang Y, Hunt N, Cox T. Adapting Narrative Exposure Therapy for Chinese earthquake survivors: a pilot randomised controlled feasibility study. BMC Psychiatry. 2014;14:262.

25. Robjant K, Fazel M. The emerging evidence for Narrative Exposure Therapy: a review. Clin Psychol Rev. 2010;30(8):1030-9.

26. Neuner F, et al. Treatment of posttraumatic stress disorder by trained lay counselors in an African refugee settlement: a randomized controlled trial. J Consult Clin Psychol. 2008;76(4):686-94.

27. Foa EB, et al. Randomized trial of prolonged exposure for posttraumatic stress disorder with and without cognitive restructuring: outcome at academic and community clinics. J Consult Clin Psychol. 2005;73(5):953-64.

28. Jacob N, et al. Dissemination of psychotherapy for trauma spectrum disorders in postconflict settings: a randomized controlled trial in Rwanda. Psychother Psychosom. 2014;83(6):354-63.

29. Gillespie K, et al. Community based cognitive therapy in the treatment of post-traumatic stress disorder following the Omagh bomb. Behav Res Ther. 2002;40(4):345-57.

30. Cusack K, et al. Psychological treatments for adults with posttraumatic stress disorder: a systematic review and meta-analysis. Clin Psychol Rev. 2016;43:128-41.

31. Gwozdziewycz N, Mehl-Madrona L. Meta-analysis of the use of Narrative Exposure Therapy for the effects of trauma among refugee populations. Perm J. 2013;17(1):70.

32. Jacob N, Wilker S, Isele D. Narrative Expositionstherapie zur Behandlung von Traumafolgestörungen. Swiss Arch Neurol Psychiatry Psychother. 2017; 168(4):99-106

33. Adenauer $\mathrm{H}$, et al. Narrative Exposure Therapy for PTSD increases top-down processing of aversive stimuli-evidence from a randomized controlled treatment trial. BMC Neurosci. 2011;12:127.

34. Hanemann WM. Willingness to pay and willingness to accept - how much can they differ? Am Econ Rev. 1991;81(3):635-47.

35. Le QA, et al. Cost-effectiveness of prolonged exposure therapy versus pharmacotherapy and treatment choice in posttraumatic stress disorder (the optimizing PTSD treatment trial): a doubly randomized preference trial. J Clin Psychiatry. 2014;75(3):222-30.

36. Morey E, Thacher JA, Craighead WE. Patient preferences for depression treatment programs and willingness to pay for treatment. J Ment Health Policy Econ. 2007;10(2):73-85

37. Unutzer J, et al. Willingness to pay for depression treatment in primary care. Psychiatr Serv. 2003;54(3):340-5.

38. Goffman E. Stigma: notes on the management of spoiled identity. Reissue edition ed. New York: Simon \& Schuster Inc; 2009.

39. Read J, et al. Prejudice and schizophrenia: a review of the 'mental illness is an illness like any other' approach. Acta Psychiatr Scand. 2006;114(5):303-18.
40. Mittal D, et al. Stigma associated with PTSD: perceptions of treatment seeking combat veterans. Psychiatr Rehabil J. 2013;36(2):86-92.

41. Lauber C, Rossler W. Stigma towards people with mental illness in developing countries in Asia. Int Rev Psychiatry. 2007;19(2):157-78.

42. Nietlisbach $\mathrm{G}$, Maercker $\mathrm{A}$. Effects of social exclusion in trauma survivors with posttraumatic stress disorder. Psychol Trauma. 2009;1(4):323-31.

43. Corrigan P. How stigma interferes with mental health care. Am Psychol. 2004;59(7):614-25.

44. Sommer, J., et al., Associations between societal disapproval and changes in symptoms of PTSD and appetitive aggression following treatment among high-risk South African males. Manuscript submitted for publication, 2017.

45. Wagner B, et al. Social acknowledgement as a predictor of post-traumatic stress and complicated grief after witnessing assisted suicide. Int J Soc Psychiatry. 2012;58(4):381-5.

46. Sommer J, et al. Associations between societal disapproval and changes in symptoms of PTSD and appetitive aggression following treatment among high-risk South African males. Eur J Psychotraumatol. 2017;8(1):1369831.

47. International-Federation-of-Red-Cross and Red-Crescent-Societies, Emergency plan of action (EPOA) Burundi: Floods. 2014. Retrieved from https://www.google.de/url?sa=t\&rct=j\&q=\&esrc=s\&source=web\&cd=9\&ved= OahUKEwjvia3Hp4TWAhXSJIAKHSQUDp0QFghMMAg\&url= http\%3A\%2F\%2Fadore.ifrc.org\%2FDownload.aspx\%3FFileld\%3D55287\&usg= AFQjCNHc2rt-_1BpnMEFsJmFvdQ8ck6XYw

48. Bracken BA, Barona A. State of the art procedures for translating, validating and using psychoeducational tests in cross-cultural assessment. Sch Psychol Int. 1991;12(1-2):119-32.

49. Crombach A, Bambonye M, Elbert T. A study on reintegration of street children in Burundi: experienced violence and maltreatment are associated with mental health impairments and impeded educational progress. Front Psychol. 2014:5:1441.

50. Nandi C, et al. Predictors of posttraumatic stress and appetitive aggression in active soldiers and former combatants. Eur J Psychotraumatol. 2015;6:26553.

51. Augsburger $M$, et al. Appetitive aggression and adverse childhood experiences shape violent behavior in females formerly associated with combat. Front Psychol. 2015;6:1756.

52. Foa EB, et al. The validation of a self-report measure of posttraumatic stress disorder: the posttraumatic diagnostic scale. Psychol Assess. 1997;9(4):445-51.

53. Foa EB, et al. Reliability and validity of a brief instrument for assessing posttraumatic stress disorder. J Trauma Stress. 1993;6(4):459-73.

54. Ertl $\mathrm{V}$, et al. Validation of a mental health assessment in an African conflict population. Psychol Assess. 2010;22(2):318-24

55. Hecker T, et al. Aggression inoculates against PTSD symptom severity - insights from armed groups in eastern DR Congo. Eur J Psychotraumatol. 2013;4:2070.

56. Foa EB, Tolin DF. Comparison of the PTSD symptom scale-interview version and the clinician-administered PTSD scale. J Trauma Stress. 2000;13(2):181-91.

57. Kroenke K, Spitzer RL, Williams JB. The PHQ-9: validity of a brief depression severity measure. J Gen Intern Med. 2001;16(9):606-13.

58. Nandi $C$, et al. Predicting domestic and community violence by soldiers living in a conflict region. Psychol Trauma. 2017;9:663.

59. Sheehan DV, et al. The Mini-International Neuropsychiatric Interview (M.I.N.I): the development and validation of a structured diagnostic psychiatric interview for DSM-IV and ICD-10. J Clin Psychiatry. 1998;59(20):22-33.

60. Eytan A, et al. Transcultural validity of a structured diagnostic interview to screen for major depression and posttraumatic stress disorder among refugees. J Nerv Ment Dis. 2007:195(9):723-8.

61. Vogel DL, Wade NG, Ascheman PL. Measuring perceptions of stigmatization by others for seeking psychological help: reliability and validity of a new stigma scale with college students. J Couns Psychol. 2009;56(2):301-8.

62. Blais RK, Renshaw KD. Stigma and demographic correlates of helpseeking intentions in returning service members. J Trauma Stress. 2013; 26(1):77-85.

63. Blais RK, Renshaw KD, Jakupcak M. Posttraumatic stress and stigma in active-duty service members relate to lower likelihood of seeking support. J Trauma Stress. 2014;27(1):116-9.

64. Corp, I. IBM SPSS statistics for windows. Armonk: IBM Corp; 2011.

65. R-Development-Core-Team. R: a language and environment for statistical computing. Vienna: R Foundation for Statistical Computing; 2008.

66. Jacobson NS, Truax P. Clinical significance: a statistical approach to defining meaningful change in psychotherapy research. J Consult Clin Psychol. 1991; 59(1):12-9. 
67. Kolassa I-T, et al. Spontaneous remission from PTSD depends on the number of traumatic event types experienced. Psychol Trauma. 2010;2(3): 169-74.

68. Staub E. Breaking the cycle of genocidal violence: healing and reconciliation. In: Harvey JH, editor. Death, dying, and bereavement. Perspectives on loss: a sourcebook. Philadelphia: Brunner/Mazel; 1998. p. 231-8.

69. Schauer E, et al. Narrative Exposure Therapy in children: a case study. Intervention. 2004;2(1):18-32.

70. Stotz SJ, et al. The relationship between trauma, shame, and guilt: findings from a community-based study of refugee minors in Germany. Eur J Psychotraumatol. 2015;6:25863.

71. Neuner F, et al. A comparison of Narrative Exposure Therapy, supportive counseling, and psychoeducation for treating posttraumatic stress disorder in an African refugee settlement. J Consult Clin Psychol. 2004;72(4):579-87.

Ready to submit your research? Choose BMC and benefit from:

- fast, convenient online submission

- thorough peer review by experienced researchers in your field

- rapid publication on acceptance

- support for research data, including large and complex data types

- gold Open Access which fosters wider collaboration and increased citations

- maximum visibility for your research: over $100 \mathrm{M}$ website views per year

At BMC, research is always in progress.

Learn more biomedcentral.com/submissions 